\title{
Estrategia de Enseñanza Basada en el Cambio Conceptual para la Transformación de Ideas Previas en el Aprendizaje de las Ciencias
}

\author{
Mirna C. Mahmud y Oscar A. Gutiérrez \\ Universidad Pedagógica Experimental Libertador, Instituto Pedagógico de Barquisimeto Luis \\ Beltrán Prieto Figueroa, Coordinación de Postgrado. 3er Piso. Edificio Este. Final Av. Vargas, \\ Barquisimeto-Venezuela (e-mail: mahmudlic@yahoo.es y ogutier@cantv.net)
}

Recibido Nov. 12, 2009; Aceptado Dic. 14, 2009; Versión Final recibida 15 Ene. 2010

\begin{abstract}
Resumen
El objetivo de esta investigación es determinar la efectividad de estrategias didácticas basadas en el cambio conceptual para la transformación de las ideas previas sobre calor y temperatura en estudiantes universitarios de carreras relacionadas con la enseñanza de las ciencias. El diseño de la investigación consistió en aplicar una prueba inicial sobre ideas previas relacionadas con los conceptos de calor y temperatura. Luego se realizaron actividades instruccionales basadas en el modelo del cambio conceptual y finalmente se aplicó una nueva prueba para evaluar el logro de los aprendizajes. El análisis estadístico descriptivo de los itemes de las pruebas revela que hubo ligeros cambios en las ideas previas. Por otra parte, los resultados de la aplicación del estadígrafo t-de Student permiten concluir_que la estrategia basada en el cambio conceptual es efectiva para la mejor comprensión de los conceptos de calor y temperatura.
\end{abstract}

Palabras claves: cambio conceptual, enseñanza de la ciencia, calor y temperatura, conceptos erróneos

\section{Teaching Strategy Based on Conceptual Change for the Transformation of Misconceptions in Learning Science}

\begin{abstract}
The objective of this research is to determine the effect of the didactic strategies based on conceptual changes for the transformation of misconceptions about heat and temperature in College students of careers related to science. The design consisted of applying an initial test on previous ideas related to the concepts of heat and temperature. Then, a learning experience with instructional activities based on conceptual change model was done to then apply a new test to assess to evaluate the new learning. The descriptive statistical analysis of the items before and after treatment revealed that there were slight changes on misconceptions. Moreover, the results of applying the t-Student statistic suggest that the strategy based on conceptual changes is effective for the better comprehension of the concepts of heat and temperature.
\end{abstract}

Keywords: conceptual change, teaching of science, temperature, misconceptions 


\section{INTRODUCCIÓN}

Investigaciones muestran que los estudiantes no vienen a clase con una mente en blanco. Generalmente ellos tienen ideas propias para interpretar los fenómenos de la naturaleza, el mundo que los rodea En algunos casos, estas concepciones concuerdan con los nuevos conocimientos enseñados en clase, pero a veces existen contradicciones entre sus creencias que no coinciden con las ideas científicamente aceptadas. Así mismo estos conocimientos previos influyen en como los estudiantes aprenden el nuevo conocimiento científico. Diversos autores establecen que es necesario que el docente conozca las ideas que tienen los estudiantes sobre un tema en particular ya que ellas influirán en el aprendizaje, como lo afirmó Ausubel (2002) en su famosa frase "Si tuviera que reducir toda la Psicología educativa a un solo principio enunciaría este: El factor más importante que influye en el aprendizaje es lo que el alumno ya sabe. Averígüese esto y enséñese consecuentemente".

La presencia de estas ideas de los alumnos es muy relevante para el proceso de construcción del conocimiento que llevan a cabo, dado que los estudiantes, aprenden sobre la base de lo que ya conocen. Al incorporar una nueva información, activan en su memoria los conocimientos relacionados con ella, establecen conexiones e interpretan la nueva información en función del conocimiento previo existente, (Ausubel et al,1983). Los investigadores han dado diferentes denominaciones a estas concepciones previas, que dependen de la tendencia del investigador: ideas intuitivas, ciencia de los niños, representaciones de los alumnos (Osborne, 1983), Errores conceptuales (Helm, 1980), Preconcepciones (Novak, 1983), Concepciones alternativas, o marcos alternativos (Driver, 1978) que después denominaron ideas de los niños, representaciones (Giordan,1982) y preconceptos (Mc Dermott,1984 y Duit,1984). Cada una de estas denominaciones lleva consigo implicaciones teóricas y una connotación del enfoque perteneciente a los estudios que se realizaron, pero de manera general se refieren al mismo planteamiento, coinciden en las características y definiciones que presentan acerca de ellas, concibiéndolas como elementos determinantes en el aprendizaje y la enseñanza en las Ciencias.

Generalmente, en la literatura de investigación educativa, especialmente en la Didáctica de las ciencias se utilizan predominantemente los términos ideas previas y preconcepciones. En este trabajo se utilizará la acepción de ideas previas definidas como el conjunto de ideas que poseen los seres humanos para la interpretación de los fenómenos naturales, y que las mismas están en contradicción con lo establecido en las teorías, principios y leyes del conocimiento científico o paradigmas predominantes en el medio académico. Son el fruto de la percepción y estructuración cognitiva basadas en experiencias cotidianas tanto físicas como sociales que dan como resultado un conocimiento empírico de la ciencia (Pozo y Gómez, 2001; Moreira y Greca, 2005).

Desde la década de los ochenta ha surgido un interés en la investigación de las ideas previas sobre calor y temperatura. Diferentes estudios han demostrado que los estudiantes tienen dificultades en la comprensión de los conceptos de calor y temperatura y tienen diferentes ideas a aquellas de las sostenidas por los científicos (Cervantes, 1987 y Aloma y Malaver, 2007). En la enseñanza de las ciencias, el tópico de calor y temperatura ocupa un lugar importante, ya que tienen relación en el aprendizaje de otros contenidos tales como entalpía. Además son conceptos que históricamente tiene problemática en su enseñanza, y por lo tanto se han realizado numerosas investigaciones relacionadas con las ideas previas o preconcepciones de los mismos. En la comunidad científica se acepta el enfoque cinético molecular dinámico donde se define el concepto de calor como una transferencia de energía entre el sistema y su entorno debido a una diferencia de temperatura, es decir el calor está definido en término de un proceso, tal como lo plantea Levine, 1995 (citado en Domínguez, 2007). En cuanto al concepto de temperatura diremos que es una magnitud intensiva, relacionada directamente con la energía cinética molecular media de las partículas y, en consecuencia, con la agitación de las mismas. En otras investigaciones Díaz (1996) reporta algunas expresiones sobre el calor, tales como: "es el sol", "es lo que producen los rayos del sol", "es el fuego", "es cuando un objeto está caliente", "es lo opuesto al frío". Por su parte, González (1998) analiza algunas concepciones previas sobre calor y temperatura, las cuales desde el punto de vista termodinámico son incoherentes: "cierra la ventana para que no entre frío", "el calor es algo caliente", "llegó el frío de diciembre" y "los rayos del sol están calientes". 
Bacas (1997) publicó los resultados de estudios sobre preconceptos de calor y temperatura concluyendo que el uso incorrecto de la palabra calor en la vida cotidiana y las confusiones entre los conceptos calor y temperatura es lo que propicia en los estudiantes la formación de preconcepciones cuyo significados son diferentes a la los científicos. Asimismo, Domínguez et al. (1998) presentan una compilación de investigaciones de ideas previas o preconcepciones sobre calor y temperatura. Ellos las denominan ideas alternativas, como se presentan en la Tabla 1. En el estudio realizado por Domínguez (1998) entre estudiantes de 12 a 23 años sobre las ideas alternativas relacionados con los conceptos de calor y temperatura determinó que un número alto de estudiantes tiene concepciones previas erradas, tales como "el cuerpo tiene Calor" y "temperatura es calor". Concluye que persiste la influencia del lenguaje cotidiano en la utilización y verbalización de gran parte de las ideas referidas al calor y temperatura.

En un estudio transversal realizado por Domínguez (1998) entre estudiantes de 12 a 23 años sobre las ideas alternativas relacionados con los conceptos de calor y temperatura determinó que un número alto de estudiantes tiene concepciones previas erradas, tales como "el cuerpo tiene Calor" y "temperatura es calor". Concluye que persiste la influencia del lenguaje cotidiano en la utilización y verbalización de gran parte de las ideas referidas al calor y temperatura.

Tabla 1: Algunas Ideas alternativas sobre calor y temperatura (Domínguez et al., 1998).

\begin{tabular}{|l|l|}
\hline Conceptos & Ideas Alternativas \\
\hline Calor & $\begin{array}{l}\text { Algo material, contenido en el cuerpo (sistema); cuanto más calor tiene el cuerpo más } \\
\text { caliente estará. } \\
\text { En los cuerpos el calor puede pasar de unas partes a otras o de unos cuerpos a otros. }\end{array}$ \\
\hline Calor / Frío & $\begin{array}{l}\text { Son dos fluidos materiales y opuestos. La sensación calor/frío es consecuencia de la } \\
\text { transferencia del calor/frío al cuerpo. }\end{array}$ \\
\hline Calentar / Enfriar & Ganancia o pérdida de ese ente material llamado calor \\
\hline Caliente / Frío & $\begin{array}{l}\text { Son propiedades características de los cuerpos. Por ejemplo, los metales son fríos por } \\
\text { naturaleza. }\end{array}$ \\
\hline Temperatura & $\begin{array}{l}\text { Temperatura = calor. Temperatura y calor son sinónimos y aquélla, en todo caso, mide } \\
\text { la cantidad de calor que tiene el sistema. } \\
\text { La temperatura depende de la masa o del volumen. }\end{array}$ \\
\hline Dilatación & $\begin{array}{l}\text { Paso de calor al interior del cuerpo haciéndolo más grande y, como consecuencia, } \\
\text { más pesado. }\end{array}$ \\
\hline
\end{tabular}

Bañas et al, (2003) realizaron un diagnóstico y análisis en estudiantes universitarios sobre las concepciones calor y temperatura. Entre las principales ideas encontraron que los estudiantes no consideran constante la temperatura durante los cambios de estados, que la temperatura aumenta proporcionalmente con la masa, y la mayoría tienen la idea que el calor es una forma de energía y no interpretan el concepto como un proceso de transferencia de energía. Lo consideran como una propiedad de los cuerpos y no un mecanismo. Se otorga a la temperatura una propiedad extensiva y un pequeño porcentaje tiende a confundir calor con temperatura. Estos resultados concuerdan con lo reportado en la literatura con la presencia de las ideas previas.

Por otra parte, Aloma y Malaver (2007) analizaron los conceptos de calor, trabajo, energía y del teorema de Carnot en libros universitarios de termodinámica usando técnicas de análisis de contenido de texto. En muchos de los libros analizados presentan conceptos con características que corresponden a concepciones espontáneas o no formales, lo que podría generar interpretaciones erróneas en el estudiante que se inicia en un curso de termodinámica. Aun cuando muchos de los textos de la muestra presentan el concepto de calor como una forma de transferencia de energía, en todos los textos analizados aparecen frases que confunden el calor como una forma de energía o lo definen como energía que se transfiere. Indudablemente, calor y temperatura son entre los conceptos más difíciles de la enseñanza y aprendizaje de la ciencia y por lo tanto es importante realizar investigaciones sobre las ideas previas y desarrollar estrategias didácticas para estimular el cambio de esas ideas previas y promover un aprendizaje significativo. 
Los investigadores coinciden en la existencia de las ideas previas que tienen todos los individuos, pero no hay acuerdo sobre el origen de las mismas. Entre las causas de las ideas previas, (Pozo,1996) señala las experiencias y observaciones de la vida cotidiana, el profesorado, los libros de texto y otros materiales escolares, la interferencia del lenguaje cotidiano y el científico, los medios de comunicación, y la cultura propia de cada civilización. Por otra parte, Lin et al, (2004) afirman que el origen de las preconcepciones está en: (a) la enseñanza dentro y fuera de la escuela; (b) las experiencias diarias cotidianas, (c) el medio social y (d) la intuición. En cambio, Pozo et al (1991) clasifican las preconcepciones o ideas previas de acuerdo con un origen sensorial, cultural y escolar. El sensorial origina las concepciones espontáneas como consecuencia del intento de dar significado a las actividades cotidianas y serían motivadas por los procesos sensoriales y perceptivos. Ante toda situación nueva o problemática se inicia la búsqueda de información que permita predecir y controlar ese suceso; esa búsqueda de información no es sistemática y rigurosa sino que se realiza de forma simplificada y cómoda.

En el origen cultural, las ideas surgirían como consecuencia del contacto con el entorno cultural y social del alumnado quien accede a las aulas con un importante bagaje fruto de su entorno y proceso de socialización. Todas estas creencias originan las concepciones sociales; y en la sociedad actual la abundancia de información científica proveniente de los medios de comunicación y el entorno del alumnado constituyen un bombardeo difícil de interpretar y entender. Además de los dos anteriores, el origen escolar es una fuente importante de ideas previas, las concepciones análogas. Tanto la existencia de errores provenientes del profesorado, de los materiales o de la propia interpretación del alumnado, como las presentaciones deformadas o simplificadas llevan a una comprensión errónea de ciertos conceptos o principios Independiente de su origen, las ideas previas se caracterizan por ser universales, coherentes, persistentes, y consistentes. La universalidad se refiere a que los estudiantes muestran concepciones similares 0 parecidas, en diferentes países o culturas, y en edades similares. En la coherencia, los esquemas que poseen los alumnos se caracterizan por el nivel de organización, es decir, por las relaciones que se establecen entre los conocimientos que integran y por el grado de interconexión lógica. La estabilidad de tales ideas y la resistencia a la instrucción es la persistencia, mientras que la consistencia es la utilización de una misma idea previa en contextos distintos.

Ante la realidad de la influencia de las preconcepciones en el aprendizaje de la ciencias, en las últimas décadas se han postulados diferentes modelos educativos que tienen como propósito fundamental lograr el cambio o transformación de las ideas previas de los estudiantes en concepciones aceptadas por la comunidad científica. Estos modelos se engloban bajo la denominación de cambio conceptual. La mayoría de los investigadores concuerdan en describir el cambio conceptual como un proceso de aprendizaje donde el estudiante modifica sus concepciones sobre un fenómeno o principio mediante la reestructuración o integración de la nueva información en sus esquemas mentales preexistentes.

En la literatura de Didáctica de las Ciencias Naturales, la expresión "enseñanza por cambio conceptual" se refiere a la aplicación de estrategias instruccionales que (a) tomen en cuenta el conocimiento previo y experiencias del estudiante, (b) identifiquen preconcepciones comunes, (c) orienten la planificación de actividades mas adecuadas para el entendimiento de los conceptos en ciencia y (d) estimulen al estudiante a modificar o crear una estructura cognitiva para el nuevo conocimiento modificado o construido Posner et al, (1982) elaboraron un modelo de cambio conceptual fundamentado en las nociones de acomodación y de desequilibrio de la teoría de Piaget: en las ideas de Kuhn acerca de las revoluciones científicas; así como en los postulados del" Programa de Investigación" de Lakatos con su noción de núcleo central. En su modelo, Posner establece que existe paralelismo entre el desarrollo conceptual de un individuo y la evolución histórica de los conceptos científicos, y que el cambio se produce por la presencia de conflictos cognitivos. En este modelo, se necesitan cuatro condiciones para que se produzca el cambio conceptual: a). La nueva concepción debe ser potencialmente fructífera. Debe resolver problemas actuales o responder preguntas a las nuevas situaciones; b). Debe existir una insatisfacción con las concepciones existentes. Sí las ideas y conocimientos que posee el individuo son satisfactorias para la comprensión de un determinado fenómeno, es poco probable que acepte una nueva concepción; c). El estudiante debe ser capaz de entender lo que significa la misma, Es decir la nueva concepción debe ser inteligible; y d) d). La nueva concepción debe ser 
consistente con el conocimiento existente aunque inicialmente contradiga las ideas previas del alumno. (Concepción plausible).

Por lo tanto, la enseñanza y aprendizaje bajo este modelo exige que los docentes en su planificación metodológica deban tomar en cuenta tanto las preconcepciones de los alumnos como las estrategias instruccionales que favorezcan la creación de conflictos entre las ideas previas y las nuevas ideas científicas.

Se han propuestos diversos enfoques de estrategias instruccionales basados en el modelo de cambio conceptual de Posner. Así, Soussan, 1982 (citado en Nieda y Macedo,1997) sugiere un enfoque que consta de los siguientes momentos de aprendizaje: (a) acercamiento, (b) expresión de las ideas previas, (c) búsqueda, (d) movilización, (e) estructuración, (f) refuerzo y (g) transferencia. En conjunto, estos momentos sirven de lineamientos generales de tal modo que el docente planifique sus estrategias instruccionales dirigidas al logro de cambios conceptuales en las preconcepciones de los alumnos. En resumen, la aplicación del modelo de cambio conceptual involucra descubrir las preconcepciones de los estudiantes acerca un tópico o fenómeno y usar diferentes estrategias instruccionales para ayudar a modificar su estructura cognoscitiva, y por ende la transformación de sus preconcepciones. Por otra parte investigaciones de Jasien y Oberem, (2002) han mostrado que no solamente los estudiantes sino que docentes en ejercicio así como futuros docentes estudiantes de carreras universitarias también tiene ideas previas en contenidos de ciencia como Biología, Química y Física. Los hallazgos de estas investigaciones sugieren la preocupación de que sí estos futuros docentes tienen ideas previas sobre contenidos de la ciencia, entonces habrá la posibilidad de que transmitiesen las mismas ideas erróneas a sus futuros alumnos en la actividades de aula. Basado en lo anterior, se planteó la necesidad de indagar sobre la efectividad de estrategias instruccionales que contribuyan a modificar las ideas previas en estudiantes universitarios de carreras docentes. Por lo tanto, el objetivo de experiencia educativa fue determinar si estrategias basadas en el cambio conceptual podrían transformar las ideas previas sobre calor y temperatura en estudiantes universitarios de carreras docentes relacionadas con la enseñanza de las ciencias.

\section{METODOLOGÍA}

Diseño la investigación: El estudio se realizó bajo el paradigma cuantitativo en la modalidad de investigación de campo. El diseño de la investigación consistió en aplicar una preprueba sobre ideas previas relacionadas con los conceptos de calor y temperatura. Luego se realizó una experiencia didáctica con actividades instruccionales basadas en el modelo del cambio conceptual y finalmente se aplicó una postprueba para evaluar el logro de los aprendizajes de los estudiantes.

Sujetos de investigación: Los sujetos de estudio fueron 20 estudiantes de ciencia de una Universidad dirigida a la formación de docentes para la educación media.

Instrumentos: Se utilizó el instrumento denominado Prueba sobre ideas previas de calor y temperatura con el propósito de obtener información acerca de las preconcepciones que tienen los estudiantes sobre los conceptos de calor y temperatura- Esta prueba, aplicada antes y después del tratamiento está formada por un conjunto de ítems de selección simple. Además el estudiante debe explicar o justificar la opción seleccionada entre las diferentes planteadas en el ítem. Cada pregunta planteaba una situación sobre calor o temperatura seguida por afirmaciones sobre ideas previos comunes así como la afirmación correcta aceptada como conocimiento válido en la comunidad científica. En la tabla 2 aparece una selección de algunas de las preguntas.

Diseño instruccional: El tratamiento experimental denominado Estrategia Didáctica de Cambio Conceptual, fue aplicado a los estudiantes universitarios con el propósito de evaluar el efecto en sus preconcepciones de Calor y Temperatura.

El enfoque metodológico del cambio conceptual plantea la necesidad de que los profesores conozcan las ideas previas de los alumnos y a su vez las empleen para favorecer la creación de conflictos cognitivos entre las ideas espontáneas y las científicas. En ese sentido, los objetivos planificados se aplicaron en tres sesiones de clase, desarrolladas en tres horas por sesión, contentivas en diferentes actividades, eficaces para la participación activa y creativa de los estudiantes y del docente, estableciendo la interacción alumno-alumno y docente-alumno, necesario para el logro de la estrategia aplicada. En este orden de ideas, es importante señalar la 
posición que debe asumir el docente para la estrategia didáctica, que se basa en realizar anotaciones acerca de los incidentes que tuvieron más relevancia, con el fin de tomar decisiones en cuanto a los cambios en la planificación y ejecución del proceso de enseñanza-aprendizaje.

Tabla 2: Algunas preguntas de la prueba sobre aplicación de ideas previas de calor y temperatura.

¿Qué es necesario para que exista calor?: a) Un solo cuerpo, b) Por lo menos dos cuerpos a diferentes temperaturas, c) Un solo cuerpo que tiene que estar "caliente, d) Ninguna de las proposiciones anteriores.

Un alumno entra al laboratorio de ciencias que está a una temperatura ambiental de $25{ }^{0} \mathrm{C}$ donde se encuentra una mesa de hierro y otra de madera. Inmediatamente enciende el aire acondicionado .Pasada dos horas la temperatura ambiental está a $20^{\circ} \mathrm{C}$. La temperatura de la mesa de hierro será: a) Menor que en la mesa de madera, b) mayor que en la mesa de madera, c) Igual que en la mesa de madera, d) ninguna de las proposiciones anteriores

¿Donde está presente el calor? : a) En cualquier cuerpo, ya que todo cuerpo posee calor, b) Solamente en aquellos cuerpos que están calientes, c) En cuerpos de diferente temperatura pero en contacto, d) Todas las proposiciones anteriores.

Un profesor en el laboratorio de Química hace la siguiente demostración: Muestra dos vasos precipitados de igual forma y tamaño. El vaso A tiene el doble volumen de agua que el vaso de precipitado B. Luego, somete a calentamiento las masas de agua hasta el punto de ebullición. Por lo tanto, la temperatura del agua en el envase $A$ será: a) mayor que la temperatura del agua del vaso $B, b)$ Igual que la temperatura del agua del vaso precipitado B, c) Menor que la temperatura del agua del vaso precipitado

Varios objetos de metal y de madera son colocados en el interior de un congelador que se encuentra a una temperatura de $-10{ }^{\circ} \mathrm{C}$. Después de 24 horas se puede deducir que la temperatura de los objetos de madera es: a) Mayor que la temperatura de los objetos de metal, b) Menor que la temperatura de los objetos de metal, c) Igual que la temperatura de los objetos de metal.

Para que se pueda admitir la existencia de calor en un cuerpo debe haber: a) una diferencia de temperatura, b) una diferencia de masa, c) una diferencia de energía, d) todas las proposiciones anteriores

Calor es: a) energía cinética de las moléculas; b) Energía transmitida solo por medio de una diferencia de temperaturas; c) La energía contenida en un cuerpo.

Dos esferas del mismo material pero cuyas masas son diferentes quedan durante mucho tiempo en un horno. Al retirarlas del horno, son inmediatamente puestas en contacto. En esa situación: a) Fluye calor de la esfera de mayor masa hacia la menor masa; b) Fluye calor de la esfera de menor masa hacia la mayor masa; c) Ninguna de las dos esferas cede calor a la otra.

Las mismas esferas de la pregunta anterior son ahora dejadas durante mucho tiempo en un congelador. En esa situación, al retirarlas e inmediatamente ponerlas en contacto: a) ninguna de las esferas posee calor debido a su baja temperatura, b) fluye calor de la esfera de mayor masa hacia la de menor masa, c) ninguna de las esferas puede ceder calor a la otra.

Este diseño tuvo como base una estrategia didáctica basada en cambio conceptual, de acuerdo al modelo de Posner et al, (1982). Esta estrategia se desarrolla a través de siete momentos o actividades que corresponden al aprendizaje constructivista.

1. Momento de motivación. Es la fase inicial que pretende motivar al alumno para la nueva situación de aprendizaje, es decir, despertar en él, una curiosidad. En esta etapa se tiene que movilizar al alumno comprometiéndole afectivamente para las nuevas actividades a realizar. Para esta metódica, el docente utilizó como herramienta los conceptos de calor y temperatura, y la experiencia se llevó a cabo en el área del laboratorio, mediante la aplicación de dinámicas de grupo (Sopa de Letras y Sopa Gráfica), planificadas para cada encuentro. Además, el docente con el fin de crear el ambiente y los recursos adecuados, se reunió con el grupo en su totalidad una semana antes del comienzo de las actividades a desarrollar, por lo que los estudiantes estaban equipados con información de apoyo y materiales escolares.

2. Momento de expresión de las ideas previas. A través de la interacción docente-alumno y alumno-alumno permite identificar los contenidos conceptuales que tienen los alumnos con respecto al nuevo contenido. Por tanto, se presentó a los participantes situaciones problemáticas y preguntas, para que dieran explicaciones que permitieran detectar cuáles son las ideas previas 0 teorías que maneja, y a medida que iban emergiendo se anotaban en el pizarrón frases, palabras 
u oraciones cortas. Estas posteriormente fueron utilizadas en otros momentos de la estrategia. Además, para esta dinámica se suministró a los estudiantes materiales de apoyo relativo al contenido en estudio (artículos de material impreso con contenido relacionado con los conceptos de calor y temperatura) y fuentes de información que los mismos alumnos llevaron previa notificación.

3. Momento de búsqueda. Resolver una situación propuesta por el docente para buscar en el alumno información de sus conocimientos de los tópicos tratados en la estrategia, la cual variará según el tipo de situación: bibliográfica, experimentos, intervención del profesor y audiovisual. Para esta actividad se realizó una demostración práctica para promover los nuevos conceptos calor y temperatura, induciendo a los estudiantes que interpretaran lo observado, a través del planteamiento de interrogantes que fueron respondidas por los participantes. El docente hizo anotaciones de las respuestas que posteriormente se utilizaron para el siguiente momento.

4. Momento de movilización y conflicto. Busca con ello provocar en los estudiantes por medio de una situación (experimento, pregunta, afirmación, etc.), la confrontación entre las nuevas explicaciones y las preexistentes provocando un conflicto socio-cognitivo. En referencia a lo anterior, debe producirse insatisfacción con las ideas existentes, es decir, que frente a determinadas situaciones concretas las ideas previas resulten insuficientes o no sean útiles para interpretar las situaciones observadas o desarrolladas en el aula de clase. Con base en las preguntas y respuestas que plantearon los alumnos, el docente va aclarando las dudas y promueve la comprensión de los nuevos conocimientos. Este intercambio entre todos los involucrados en el proceso de enseñanza-aprendizaje, es lo que permite que los estudiantes se sientan en la libertad de predecir, explicar y por tanto llegar a resolver los problemas eficientemente.

5. Momento de estructuración. La existencia de las nuevas explicaciones, supone que los alumnos han establecido modificación en los conocimientos almacenados en las estructuras mentales. Para la actividad se retomaron las ideas previas anteriormente expuestas, las notas en el momento de la experiencia realizada, que aunada a las respuestas planteadas por los estudiantes a las interrogantes del docente, lo que permitió entre todos estructurar los nuevos conocimientos. Ante la situación planteada, la confrontación por parte de los estudiantes con las nuevas ideas, supone que estos han establecido distintas concepciones conceptuales a las que traían antes de la experiencia con la estrategia de cambio conceptual, lo que supone que los alumnos originaron nuevas estructuras mentales.

6. Momentos de refuerzo y transferencia. Afianzar lo aprendido y transferir las recientes adquisiciones mentales es parte del proceso de la estrategia de cambio conceptual; y en consecuencia deben presentarse a los alumnos actividades que los fuercen a aplicar las nuevas estructuras adquiridas. En este sentido, tales estructuras son al principio muy frágiles y es preciso consolidarlas. Para este momento, la investigadora extrapola con un material de apoyo la importancia del contenido aprendido, no solo para las actividades escolares sino también para las experiencias la vida cotidiana. Aunado a esto, los estudiantes realizaron carteles sobre las actividades realizadas y la interpretación de la demostración práctica en el momento de movilización y conflicto. Estos carteles fueron de gran ayuda en el momento de la evaluación.

7. Momento de evaluación. El docente orienta la verificación del nuevo conocimiento y de las actividades desarrolladas en una interacción docente-alumno y alumno-alumno. Para el término de la estrategia se realizó una exposición en forma grupal, de los carteles elaborados con los conceptos de calor y temperatura. Para ello se contó con notas de las respuestas planteadas por el grupo antes y después de la estrategia, material de apoyo aportado por el docente y los estudiantes, y las interpretaciones de los experimentos realizados. En relación a esto último, todos los estudiantes evaluaron las actividades antes y después de la aplicación de la estrategia de Cambio Conceptual, para lo cual se elaboró una pancarta que posteriormente permitió intercambiar opiniones entre los participantes involucrados (docente y participantes). Con respecto, a los siete momentos de actividad, cada uno constituyó un ciclo que no se agotaba al final del mismo, cada uno tuvo relación con los anteriores, a fin de lograr las modificaciones de las ideas previas. 


\section{RESULTADOS}

Los datos de los itemes de la pre y postprueba fueron tabulados, agrupados en dos categorías: calor y temperatura para establecer el porcentaje de respuesta correctas.

\section{Análisis de los itemes}

En esta sección se presenta un análisis estadístico descriptivo de los itemes para establecer el porcentaje de respuestas correctas referentes a las ideas previas sobre calor y temperatura. Los itemes se agruparon en dos categorías: calor y temperatura para analizar la variación de las respuestas correctas para cada una de estas categorías. La respuesta se consideraba como correcta si la explicación dada por el estudiante era consistente con la selección que hace de las diferentes proposiciones del ítem. Se observa que aunque hubo ganancia estadística, esta variación en porcentaje es muy pequeña lo que indica que hubo poco cambio en las ideas previas referentes al concepto temperatura. La fig. 1 resume los principales resultados que se discuten en lo que sigue.
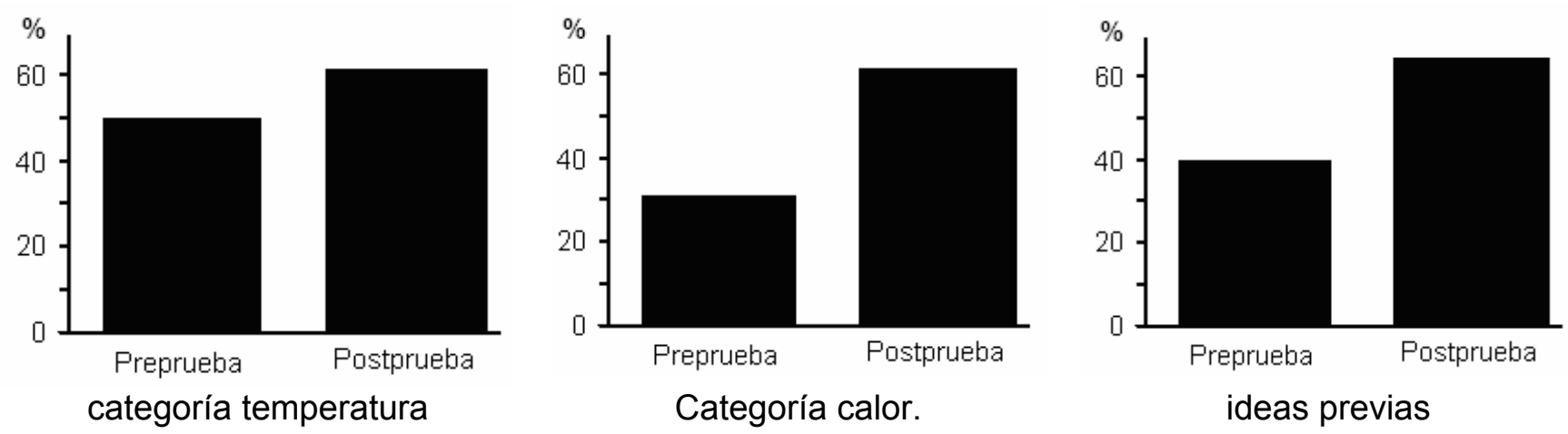

Fig.1: Porcentaje de itemes correctos en la pre y postprueba para las diversas categorías y las ideas previas de calor y temperatura.

Asimismo se analizaron las preguntas correctas antes y después de la aplicación basada en cambio conceptual, para los itemes agrupados en la categoría de calor. En la preprueba el $30 \%$ de las preguntas fueron correctas mientras que en la postprueba el porcentaje aumentó hasta un 62 $\%$. En contraste a la otra categoría temperatura, se puede observar que en esta categoría calor sí hubo un mejoramiento en el cambio de las ideas previas sobre calor.

Por ejemplo, en el siguiente ítem referente a las idea previa sobre calor: ¿Dónde está presente el calor?": a) En cualquier cuerpo, ya que todo cuerpo posee calor; b) Solamente en aquellos cuerpos que están calientes; c) En cuerpos de diferentes temperatura pero en contacto; d) Todas las proposiciones anteriores.

En la preprueba un $27 \%$ de estudiantes seleccionaron la respuesta correcta (c), y este porcentaje aumentó al $58 \%$ en la postprueba. Este aumento indica un cambio en la preconcepción sobre calor en algunos estudiantes; mientras que para el resto la idea errónea del concepto sobre calor persiste, lo cual es una de las características de las ideas previas, la persistencia de la misma. Es de hacer notar que la proposición (b) es la idea previa con mayor selección por parte de los estudiantes, lo cual indica la asociación de calor con caliente, como una cualidad y no como un proceso.

Asimismo, se efectuó un análisis estadístico descriptivo para comparar la variación en las respuestas correctas entre los itemes de la pre y postprueba. Se observa que hay diferencia en el porcentaje de respuestas correctas con un aumento para la postprueba, pero con bajo porcentaje de diferencia del $14 \%$ lo que indica que el tratamiento con la estrategia basada en el cambio conceptual tuvo influencia en la transformación de algunas ideas previas en el grupo. Sin embargo, la baja diferencia entre los porcentajes de la preprueba $(40 \%)$ y postprueba (64 \%) indica que algunas de las ideas previas persisten acorde con lo reportado en la literatura. 


\section{Efectividad del tratamiento}

Para determinar la efectividad o ganancia del tratamiento experimental se realizó un análisis con el estadígrafo $\mathrm{t}$ de Student para muestra dependientes al comparar los datos de la pre y postprueba. Los resultados se muestran en la tabla 3.

Tabla 3: Cálculo de la t de Student para muestras dependientes entre preprueba y postprueba $(\alpha=0,05)$

\begin{tabular}{|c|c|c|c|c|}
\hline $\begin{array}{c}\text { Media } \\
\text { Preprueba }\end{array}$ & $\begin{array}{c}\text { Media } \\
\text { Postprueba }\end{array}$ & $\begin{array}{c}\text { Diferencias entre } \\
\text { medias }\end{array}$ & $\begin{array}{c}\text { valor calculado } \\
\text { tp }\end{array}$ & $\begin{array}{c}\text { valor crítico } \\
\text { tc }\end{array}$ \\
\hline 4,84 & 14,56 & 9,72 & 11,9 & 2,13 \\
\hline
\end{tabular}

Se observa que hubo ganancia en la media $(9,72)$ Por otra parte, la comparación de los valores $t_{p}$ $(11,9)>t_{c}(2,13)$ permite concluir que hay ganancia estadísticamente significativa en el promedio de las calificaciones de la postprueba y la preprueba. Así, se puede concluir que hubo un cambio significativo en las transformaciones de la preconcepciones de los conceptos calor y temperatura por efecto de la aplicación de la estrategia basada en el cambio conceptual.

\section{CONCLUSIONES}

El análisis global de los resultados de los itemes permite concluir que hubo poca variación en la transformación de las ideas previas sobre calor y temperatura lo que concuerda con una de las características de las ideas previas, la resistencia al cambio ya que ellas son persistentes. Sin embargo, al comparar separadamente los itemes para las categorías calor y temperatura se observa que hubo un cambio en ambas categorías. Sin embargo hay un mayor cambio en las ideas previas referente a la categoría calor en comparación con la categoría de temperatura. Esto significa que estrategias basadas en el cambio conceptual sí ayudan al aprendizaje de los conceptos calor y temperatura.

En cambio el análisis de los calificaciones de la prueba en lo referente a las diferencias del promedio de la pre y postprueba indican que hubo ganancia estadística después de la aplicación del tratamiento; y los resultados de la t de Student señalan que hay diferencias estadísticamente significativas después del tratamiento con la estrategia de cambio conceptual. Basado en lo anterior y sí se asume que los puntajes de la preprueba y postprueba están relacionados con la comprensión del estudiante de los conceptos calor y temperatura se puede concluir que la estrategia basada en el cambio conceptual es efectiva en la transformación de las ideas previas de calor y temperatura.

La estrategia basada en el cambio conceptual es efectiva porque en la planificación de las actividades didácticas se toman en cuenta los conocimientos previos. Estas actividades permiten la confrontación de sus ideas previas con los nuevos conocimientos, se produce el conflicto cognitivo y la insatisfacción tal como se postula en los principios de la enseñanza basada en el modelo propuesto por Posner et al (1982). Al hacer relación entre sus conocimientos preexistentes y los nuevos se produce un efectivo aprendizaje significativo de acuerdo con lo planteado por Ausubel $(1983,2002)$ y por ende un mejor aprendizaje de los conceptos de calor y temperatura.

\section{REFERENCIAS}

Aloma, E. y Malaver, M. Análisis de los conceptos de energía, calor, trabajo y teorema de Carnot en textos universitarios de termodinámica. Enseñanza de las ciencias: 25(3), 387-400 (2007).

Ausubel, D. Adquisición y retención del conocimiento. Una perspectiva cognitiva. Paidos, Barcelona, España (2002).

Ausubel, D., Novak, J. y Hanesian, H. Psicología Educativa. Un punto de vista cognitivo. Trillas, México, México (1983).

Bacas, L. Detección de las ideas del profesorado acerca de los conceptos calor y temperatura. Alambique: 8, 109-116 (1997).

Bañas, C., Mellado, V. y Ruiz, C. Las ideas alternativas del alumnado de primer ciclo de Educación Secundaria Obligatoria sobre la conservación de la energía, el calor. Campo Abierto: 24, 99-126 (2003). 
Cervantes, A. Los conceptos de calor y temperatura: una revisión bibliográfica. Enseñanza de las Ciencias: 5(1), 66-70 (1987).

Díaz, A. La energía en la interacción térmica. Las ideas previas de los alumnos y alumnas. (1996) http://www.gobiernodecanarias.org/educacion/cep_sc_tenerife/recursos/revista/GAVETA/laenergia .htm.Acceso: 5 Julio (2007).

Domínguez, J., Pro, A. y García-Rodeja, E. Las partículas de la materia y su utilización en el campo conceptual de calor y temperatura: un estudio transversal. Enseñanza de las Ciencias: 16(3) 461-475 (1998).

Domínguez, J.M. (editor). Actividades para la enseñanza en el aula de ciencias. Fundamentos y planificación. 119-165, Ediciones UNL, Santa Fe, Argentina (2007).

Driver, R. Pupils and paradigms: a review of literature related to concepts development in adolescent science students. Studies in science Education: 5(1) 61-84 (1978).

Duit, R. Learning the energy concept in school-empirical results from the Phillippines and West Germany. Physics Education: 19(2) 59-66 (1984)

Giordan, A. La enseñanza de las ciencias. Siglo XXI, Madrid, España (1982)

González, O. La controversial diferencia entre Calor y Temperatura. Cenamec, Caracas, Venezuela (1998).

Helm, H. Misconceptions about physical concepts among South African pupils studying physical science. South African Journal of Science: 74, 285-290 (1980)

Jasien, P. y Oberem, G. Understanding of elementary concepts in heat and temperature among college students and K-12 teachers. Journal of Chemical Education: 79(7) 889-895 (2002).

Lin, J., Chiu, M. y Liang, J.Exploring Mental Models and Causes of Students' Misconceptions in Acids and Bases. Paper presented at the NARST(2004). Vancouver, Canada, April 1-3 (2004).

McDermot, M. Research on conceptual understanding in mechanics. Physics Today: 37(7),.24 -32. (1984).

Moreira, M. y Greca I. Cambio Conceptual: Análisis Crítico y Propuestas a la Luz de la Teoría del Aprendizaje Significativo. (2005). http://www.if.ufrgs.br/ moreira/cambioconceptual.pdf. Acceso: 30 Septiembre (2006).

Nieda, J y Macedo, B. Un Currículo Científico para Estudiantes de 11 a 14 años. Organización de Estados Iberoamericanos para la Educación, la Ciencia y la Cultura (OEI) y UNESCO, Madrid, España (1997).

Novak, J, Misconceptions in science and mathematics. Proceedings of the international Seminar. Cornelll University Press, Ithaca, New York. USA, June 20-22, (1983).

Osborne, R. Science Teaching and Children's views of the world. European Journal Science Education: 5(1), 1-14 (1983).

Posner G., Strike, K., Hewson, P. y Gertzog, W. Accommodation of a Scientific Conception: Toward a Theory of Conceptual Change. Science Education: 66(2), 211-227 (1982).

Pozo, J. Las ideas del alumnado sobre la ciencia: de dónde vienen, a donde van. y mientras tanto qué hacemos con ellas. Alambique: 7,18-26 (1996).

Pozo, J. y Gómez, M. Aprender y enseñar ciencia. Morata, Madrid, España (2001).

Pozo, J., Sanz, A., Gómez, M. y Limón, M. Las ideas de los alumnos sobre la ciencia: Una interpretación desde la Psicología Cognitiva. Enseñanza de las Ciencias: 9(1), 83-94 (1991). 